\title{
Paclitaxel induces apoptosis and reduces proliferation by targeting epidermal growth factor receptor signaling pathway in oral cavity squamous cell carcinoma
}

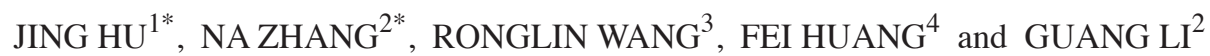 \\ ${ }^{1}$ Department of Stomatology, The Military General Hospital of Beijing PLA, Beijing 100700; ${ }^{2}$ Department of Stomatology, \\ General Hospital of The Second Artillery, Beijing 100088; ${ }^{3}$ Jinan Stomatologic Hospital, Jinan, Shandong 250001; \\ ${ }^{4}$ Department of Stomatology, Navy General Hospital, Beijing 100048, P.R. China
}

Received June 17, 2014; Accepted March 27, 2015

DOI: $10.3892 / 01.2015 .3499$

\begin{abstract}
Oral cavity cancer is common worldwide. Furthermore, the epidermal growth factor receptor (EGFR) signaling pathway is considered to be constitutively activated in oral cancers. Paclitaxel is widely accepted as an antitumor drug as it effectively inhibits the cell cycle. This study predominantly explores the possible molecule mechanism of paclitaxel on oral cancer treatment. Cell viability was first detected using an MTT assay. Cell apoptosis was examined by Hoechst staining and flow cytometry using an annexin-V and propidium iodide kit. Specific EGFR signaling pathways were further explored through western blot analysis. Abnormal protein expression levels were determined via immunofluoresence. Additionally, the protein levels of matrix metalloproteinase (MMP)-2 and 9 were determined using ELISA. Paclitaxel significantly inhibited oral cancer cell viability in a time- and dose-dependent manner. Paclitaxel also enhanced oral cancer cell apoptosis via increased Bim and Bid protein expression. Furthermore, paclitaxel was observed to inhibit oral cancer cell proliferation through increased MMP-2 and MMP-9 protein levels. Paclitaxel inhibited the growth of the oral cancer cell line, tea8113 malignant proliferation and enhanced tea8113 cell apoptosis through inhibiting the EGFR signaling pathway.
\end{abstract}

Correspondence to: Dr Fei Huang, Department of Stomatology, Navy General Hospital, 6 Fu Cheng Street, Beijing 100048, P.R. China

E-mail: huangfeipla@163.com

Dr Guang Li, Department of Stomatology, General Hospital of the Second Artillery, 16 Xinjiekou Street, Beijing 100088, P.R. China E-mail: liguangpla@163.com

*Contributed equally

Key words: paclitaxel, tea8113, epidermal growth factor receptor, oral cavity squamous

\section{Introduction}

Oral cancer is the most common malignant tumor in the oral and maxillofacial region, with an incidence rate of 0.05-0.06 per 1,000 individuals, and squamous cell carcinoma accounts for $80 \%$ of tumors of the oral cavity $(1,2)$. With the enhanced treatment options available worldwide, oral cancer treatment effect has improved to a certain degree. However, the incidence of oral cancer worldwide still increases each year (3); the incidence rate increased from 0.03 to 0.06 cases per 1,000 individuals between 2010 and 2014 (4). For oral cancer patients, the major therapies predominantly include surgery, chemotherapy and radiotherapy. However, the 5-year survival rate remains poor, at $25 \%$ (5). Thus, it is a significant challenge for clinicians to improve the efficacy of oral cancer treatment and enhance the quality of life of patients. From this perspective, to discover and develop novel and highly effective treatment methods is particularly important.

Cell division and differentiation reactions are initiated by growth factors through the cell signaling pathways that are activated by cell surface receptors. An important function of these signaling pathways is to regulate gene expression. As a crucial cell surface receptor, epidermal growth factor receptor (EGFR) has an significant role in the regulation of the cell cycle. In various types of cancer, the epidermal growth factor family of receptors is proposed to be overexpressed (6). Studies have shown that the increased expression of EGFR may regulate tumor growth, metastasis and prognosis through three major signal transduction pathways (7). Therefore, the EGFR signaling pathway may be a potential therapeutic target for oral cavity cancer patients.

The major signaling pathways of EGFR include Ras-Raf-mitogen-activated protein kinase (MAPK) (7). There are three predominant members of the MAPK family: Extracellular signal-regulated kinases (ERKs), c-Jun N-terminal kinases (JNKs) and p38 MAPKs. The Ras-Raf-MAPK pathway predominantly regulates cell survival, proliferation and differentiation by regulating the expression of various genes. ERK 1 and 2 are two subtypes of MAPK (8). The expression and distribution of ERK1/2 changes in cells, which consequently indicates the changes of the MAPK signaling pathway (9). 
ERKs are involved in the regulation of mitogen-activated proliferation/differentiation factors, such as E-cadherin, MMP-2, MMP-9, whereas JNK and p38 MAPKs are closely associated with apoptotic cell death (10). For example, the activation of JNK usually leads to the abnormal expression of cell proliferation related proteins, such as anti-apoptotic genes, BclxL and XIAP. Conversely, p38 MAPKs causes cell cycle arrest and apoptosis via a series of target genes, including p27Kip1, Bim, BclxL and XIAP (3).

Low dose paclitaxel (PTX) is a type of antitumor drug, which was originally extracted from the bark of the pacific yew tree. The pharmacological mechanism of PTX is associated with its interaction with microtubules, which are components of eukaryotic cells. Microtubules are composed of two similar polypeptide subunits ( $\alpha$ and $\beta$ ) of tubulin; PTX acts by inhibiting dynamic cytoskeletal processes and also stabilizing microtubules in cells, protecting them from disassembly and consequently blocking the progression of mitosis (11). Recent reports have shown that PTX may also inhibit cancer cell proliferation through the EGFR signaling pathway (12). The current study aims to explore the potential molecular mechanism of PTX in the treatment of oral cavity cancer.

\section{Materials and methods}

Human samples and cell lines. Human oral cancer squamous cell line tea8113 was purchased from the American Type Tissue Culture Collection (Manassas, VA, USA) and cultured in Dulbecco's modified Eage's medium (DMEM)/F12 (Hyclone, New Brunswick, NJ, USA) supplemented with $10 \%$ fetal bovine serum (Hyclone), $100 \mathrm{U} / \mathrm{ml}$ penicillin and streptomycin (Beijing Solarbio Science and Technology Co., Ltd., Beijing, China) in $25 \mathrm{~cm}^{2}$ culture flask at $37^{\circ} \mathrm{C}$ in a humidified atmosphere of $5 \% \mathrm{CO}_{2}$.

Assay of cell viability. Cell viability was determined by a colorimetric, 3-(4,5-dimethylthiazol-2-yl)-2,5-diphenyltetrazolium bromide (MTT; Sigma-Aldrich, St Louis, MO, USA) assay. In order to determine the impacts of PTX on human oral cancer squamous cell line tea8113, cells were cultured to approximately $70 \%$ confluency and starved in serum-free DMEM (SF-DMEM; Life Technologies, Inc., Carlsbad, CA, USA) overnight. Subsequently, 0.1, 1, $10 \mu \mathrm{g} / \mathrm{ml}$ PTX was pre-incubated with tea8113 cells for $48 \mathrm{~h}$. After drug treatment, the cells were cultured in fresh medium including $0.5 \mathrm{mg} / \mathrm{ml} \mathrm{MTT}$ for $4 \mathrm{~h}$. Then, DMSO was added into the wells to dissolve the blue formazan products and the density was determined spectrophotometrically (Cary 4000; Agilent Technologies, Novato, CA, USA) at a wavelength of $550 \mathrm{~nm}$. Additionally, to determine the time-dependent effects, the cells were pre-incubated with $1 \mu \mathrm{g} / \mathrm{ml}$ PTX for $12,24,48 \mathrm{~h}$ and cell viability was determined using the same method as described above. Each experiment was independently performed at least in triplicate.

Hoechst 33258 staining. Human oral cancer squamous cell line tea8113 $\left(1 \times 10^{5}\right.$ cells per well) were cultured in 6-well tissue culture plates. At 70-80\% confluence, the cells were incubated for $16 \mathrm{~h}$ in serum-free DMEM medium. Subsequently, $1 \mu \mathrm{g} / \mathrm{ml}$ PTX was added to the fresh medium and pre-incubated with the cells for $48 \mathrm{~h}$. After drug treatment, the medium was removed, and the cells were rinsed three times with cold phosphate buffered saline (PBS) and then fixed with $4 \%$ formaldehyde (Zhongshan Technology Co., Ltd., Zhongshan, China) in PBS for $20 \mathrm{~min}$ at room temperature. The cells were washed three times with cold PBS and stained with Hoechst 33258 (10 $\mu \mathrm{g} / \mathrm{ml}$; Sigma-Aldrich) for $5 \mathrm{~min}$. After staining, the cells were further washed with cold PBS and examined under a fluorescence microscope (Agilent 1200 Series Fluorescence Detector; Agilent Technologies).

Western blotting analyses. In order to conduct western blot analysis, the cells were first treated with RIPA buffer [Beijing Solarbio Science and Technology Co., Ltd.; $50 \mathrm{mM}$ Tris/HCl, $\mathrm{pH} 7.4,150 \mathrm{mM} \mathrm{NaCl} 1 \%$ (v/v) NP-40, 0.1\% (w/v) SDS] containing $1 \%(\mathrm{v} / \mathrm{v})$ phenylmethanesulfonylfluoride (Beijing Solarbio Science and Technology Co., Ltd.), 0.3\% (v/v) protease inhibitor (Sigma-Aldrich) and $0.1 \%$ (v/v) phosphorylated proteinase inhibitor (Sigma-Aldrich). The supernatants were then extracted from the lysates after centrifugation at $4,000 \mathrm{x}$ g at $4^{\circ} \mathrm{C}$ for $15 \mathrm{~min}$. To quantify the relative concentration of the total proteins, a bicinchoninic acid (BCA) protein assay kit (Pierce Biotechnology Inc., Rockford, IL, USA) was used. The proteins, at an equal amount of $15 \mu \mathrm{g}$, were separated via SDS-PAGE gel (10\% SDS (v/v) polyacrylamide) and transferred onto a polyvinylidene fluoride (PVDF) membrane at $300 \mathrm{~mA}$ for $2 \mathrm{~h}$. In order to block the non-specific binding proteins, the PVDF membrane was blocked using $8 \%(\mathrm{w} / \mathrm{v})$ milk in TBS-T for $2 \mathrm{~h}$ at room temperature. The membranes were subsequently incubated with primary monoclonal rabbit anti-human antibodies against $\beta$-actin (\#4970), p-EGFR (\#11862), EGFR (\#4405), p-ERK1/2 (\#4370), ERK1/2 (\#9102), p-JNK (\#4671), JNK (\#9252), p-p38 (\#4511), p38 (\#9212), Bcl-2 (\#2870), Bax (\#5023), p27 ${ }^{\mathrm{Kip} 1}$ (\#3686; all antibodies were purchased from Cell Signaling Technology Inc., Danvers, MA, USA) at a dilution of 1:1,000, overnight at $4^{\circ} \mathrm{C}$. The membranes were washed with PBST 4 times ( $5 \mathrm{~min} / \mathrm{time})$. Following this, horse radish peroxidase (HRP)-conjugated goat anti-rabbit IgG (Zhongshan Technology Co., Ltd.; dilution, 1:5000) was prepared and the membranes were incubated with this for $2 \mathrm{~h}$ at room temperature. After incubation, the membranes were washed 4 times (5 min/time). Subsequently, the enhanced chemiluminescence (EMD Millipore, Billerica, MA, USA) was prepared according to the manufacturer's instructions. The relative protein level was then determined. In order to qualify the changes in protein expression, the target protein was normalized against $\beta$-actin.

Apoptosis assay. To detect the effects of PTX on tea8113 cell apoptosis, the cells (50-60\% confluent) were treated with $0.1,1$, $101 \mu \mathrm{g} / \mathrm{ml}$ PTX for $48 \mathrm{~h}$. After treatment, the cells were washed with $1 x P B S$ three times. An annexin-V fluorescein isothiocyanate-propidium iodide (FITC-PI) apoptosis kit (Invitrogen Life Technologies, Inc.) was applied to determine the apoptotic rate by flow cytometry. This assay employs fluorescein-labeled annexin- $\mathrm{V}$ in conjunction with propidium iodide (PI) to detect the cells undergoing apoptosis. In summary, cells were washed with $1 \times$ PBS three times and suspended at $2-3 \times 10^{6}$ cells $/ \mathrm{ml}$ in $1 \mathrm{x}$ annexin- $\mathrm{V}$ binding buffer (10 mM HEPES/NaOH, $\mathrm{pH} 7.4$, $140 \mathrm{mM} \mathrm{NaCl}, 2.5 \mathrm{mM} \mathrm{CaCl2}$ ). Annexin-V FITC and PI buffer were added to the cells, which were then incubated at 


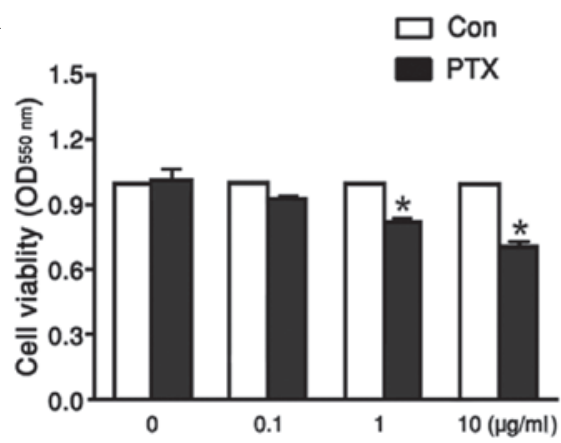

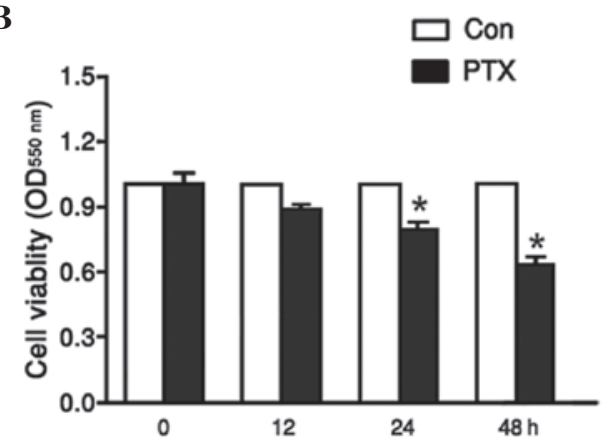

Figure 1. Tea8113 cell viability was affected by PTX in a dose- and time-dependent manner. TEA8113 cells were exposed to PTX at concentrations of $0.1,1$, and $10 \mu \mathrm{g} / \mathrm{ml}$ for $48 \mathrm{~h}$ (A). (B) TEA8113 cells were treated with $1 \mu \mathrm{g} / \mathrm{ml}$ PTX for $12,24,48 \mathrm{~h}$. Cell viability was determined by MTT assay using a spectrophotometer at $550 \mathrm{~nm}$. Data represent the means \pm standard error of the mean, $\mathrm{n}=6$ independent experiments. ${ }^{*} \mathrm{P}<0.05$ versus control. PTX, paclitaxel.

A

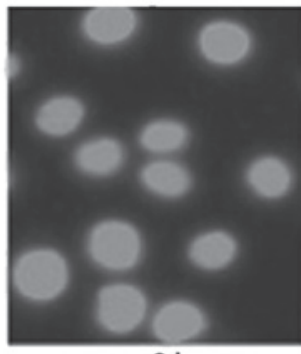

$\mathrm{Oh}$

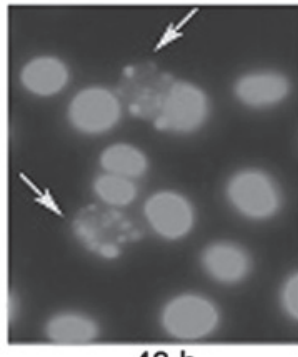

$48 \mathrm{~h}$
B

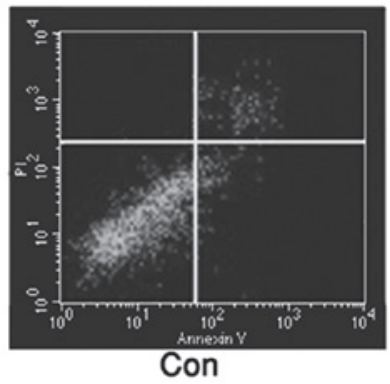

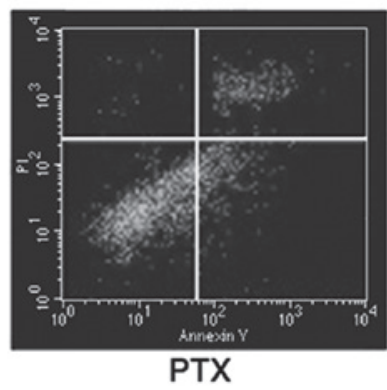

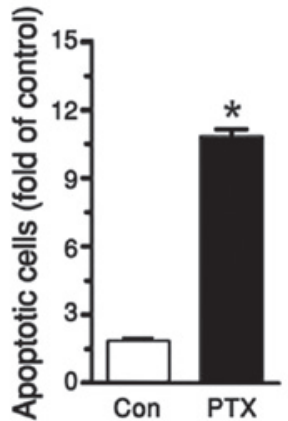

Figure 2. Enhanced TEA8113 cell apoptosis was determined when these cells were incubated with $1 \mu \mathrm{g} / \mathrm{ml}$ paclitaxel for 48 h. (A) Hoechst staining demonstrated enhanced cell apoptosis. (B) Tea8113 cell apoptosis was detected using an annexin-V and propidium iodide kit. Data represent the mean \pm standard error of the mean, $\mathrm{n}=3$ independent experiments. ${ }^{*} \mathrm{P}<0.05$ versus control.

room temperature for $15 \mathrm{~min}$ in the dark. The cells without any treatment were used as an internal control. After incubation, the cells were filtered using a filter screen and the cells were analyzed by flow cytometry (Becton Dickinson, Franklin Lakes, NJ, USA) within $1 \mathrm{~h}$ of staining using the FL1 (FITC) and FL3 (PI) lines.

Immunochemistry. The sections from BCA were used for immunohistochemistry staining to identify EGFR. Briefly, the sections were incubated with polyclonal antibodies at $37^{\circ} \mathrm{C}$ for $60 \mathrm{~min}$ or at $4^{\circ} \mathrm{C}$ overnight and labeled with HRP-conjugated anti-rabbit $\mathrm{IgG}$ at $37^{\circ} \mathrm{C}$ for $60 \mathrm{~min}$. Finally, the coverslips were mounted with DABCO and analyzed using an upright microscope (Carl Zeiss, Heidenheim, Germany). Monoclonal rabbit anti-human antibodies against EGFR (1:50 dilution) were purchased from BD Biosciences (San Jose, CA, USA) and monoclonal rabbit anti-human $\beta$-actin antibody (1:100 dilution) was purchased from Santa Cruz Biotechnology, Inc., (Dallas, TX, USA).

Immunofluorescence. Cells grown on chamber slides were washed with PBS for 15 min (total), fixed in $4 \%$ paraformaldehyde for $30 \mathrm{~min}$ at room temperature and permeabilized with $0.1 \%$ TritonX-100 at room temperature for $5 \mathrm{~min}$. After three washes with PBS for 15 min (total), non-specific binding was blocked with $3 \%$ bovine serum album (BSA) in PBS for $1 \mathrm{~h}$ at room temperature. Following this, the cells were incubated with the following primary antibodies: Human E-cadherin (\#5296) and GAPDH (\#5174; Cell Signaling Technology, Inc.,), which were all diluted at 1:100 in PBS with 1\% BSA. After the cells were incubated with primary antibodies for $2 \mathrm{~h}$ at room temperature, they were washed with PBS and 
A
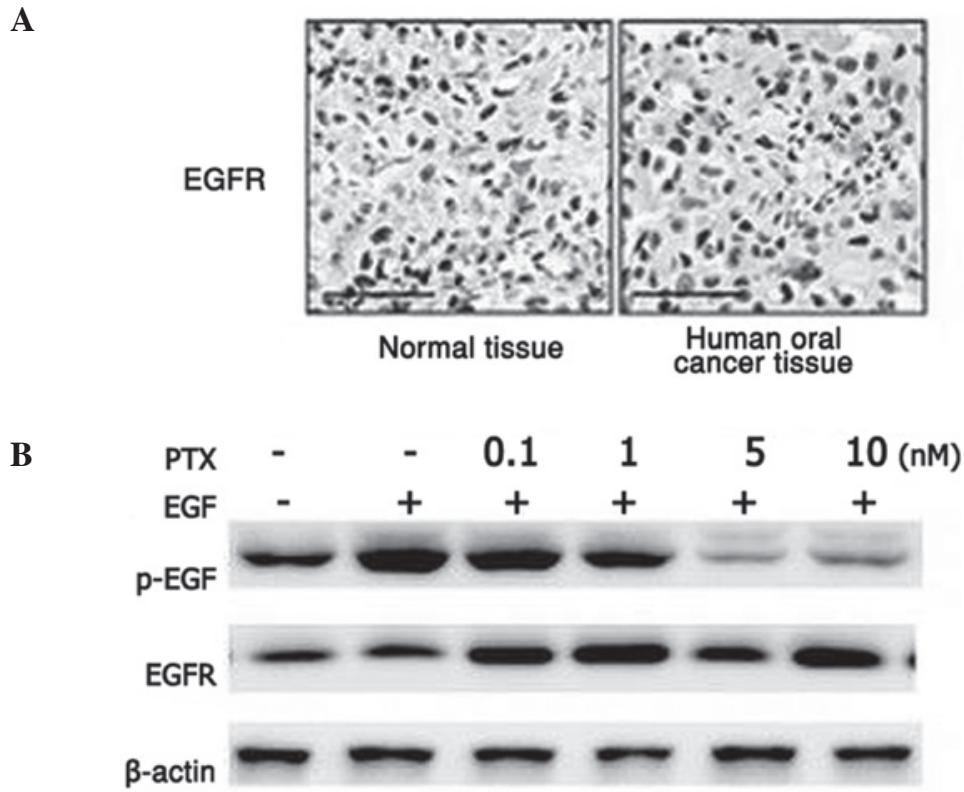

C
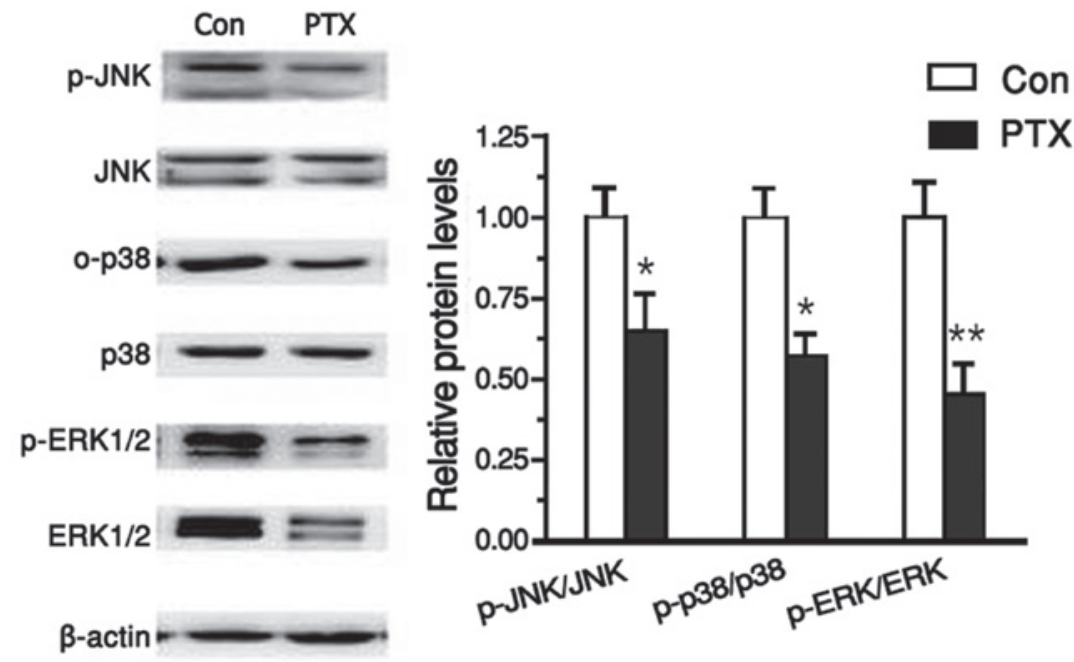

Figure 3. EGFR signaling pathway was significantly suppressed with PTX treatment. (A) Histochemistry staining demonstrated that EGFR was significantly increased in human oral cancer tissues compared with the normal tissue. (B) Western blot analysis revealed that PTX inhibited EGF-induced EGFR activation. (C) Western blot analysis demonstrated reduced c-Jun N-terminal kinases, extracellular signal-regulated kinases, p38 activation of EGFR signaling pathways. Data represent the means \pm standard error of the mean, $\mathrm{n}=3$ independent experiments. * $\mathrm{P}<0.05$ versus control. Epidermal growth factor receptor; PTX, paclitaxel.

incubated with Alexa Fluor 488-conjugated anti-rabbit IgG (ZF-0316) or TRITC-conjugated anti-mouse IgG (ZF-0313; Zhongshan Biotechnology Co., Ltd.) at 1:50 dilution in PBS with $1 \% \mathrm{BSA}$ for $1 \mathrm{~h}$ at room temperature. After several washes (15 min in total) with PBS, the cell nuclei were visualized with Hoechst 33258 staining at a concentration of $10 \mu \mathrm{g} / \mathrm{ml}$ for $10 \mathrm{~min}$ at room temperature. The slides were subsequently washed again, dried, mounted, and examined using a fluorescence microscope.

Statistical analysis. The data are presented as the mean \pm standard error of the mean (SEM). The number of independent experiments was represented by ' $n$ '. Multiple comparisons were performed using one-way analysis of variance (ANOVA) followed by Tukey's multiple-comparison test; $\mathrm{P}<0.05$ was considered to indicate a statistically significant difference.

\section{Results}

Tea8113 cell viability was affected by PTX in a dose-and time-dependent manner. To explore the effect of PTX on tea8113 cell viability, the oral cancer squamous cells were treated with PTX at concentrations of $0.1,1$, and $10 \mu \mathrm{g} / \mathrm{ml}$ for 48 h. Cell viability was then analyzed via MTT assay. As shown in Fig. 1A, tea8113 cell viability was reduced from 0.74 to 0.46 with increasing concentrations of PTX from $0-10 \mu \mathrm{g} / \mathrm{ml}$. Furthermore, tea8113 cells were treated with $1 \mu \mathrm{g} / \mathrm{ml}$ PTX for $12,24,48 \mathrm{~h}$ and the corresponding cell viability was determined by MTT assay. According to the statistics, tea8113 cell viability was decreased by 11 and $21 \%$ at 24 and $48 \mathrm{~h}$ respectively (Fig. 1B). These results suggested that tea8113 cell viability was evidently decreased by PTX in a dose- and time-dependent manner. 
A

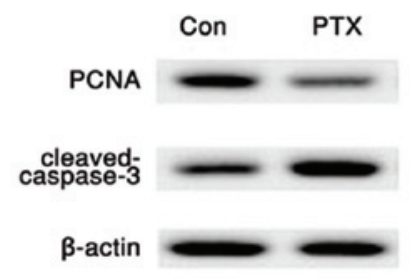

B

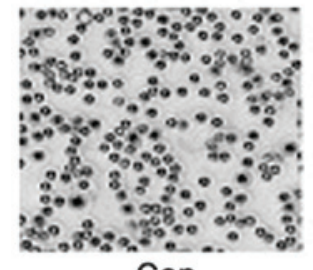

Con

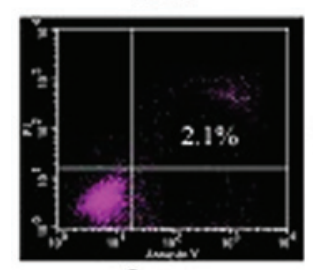

Con

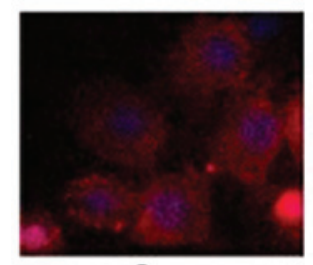

Con

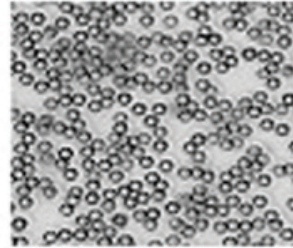

EGF

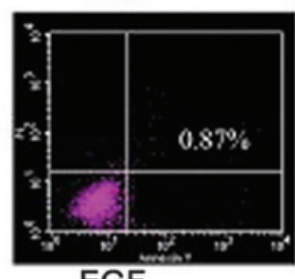

EGF

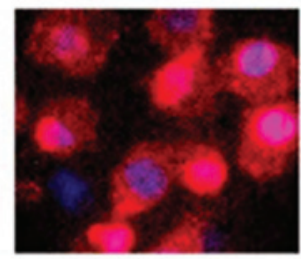

EGF

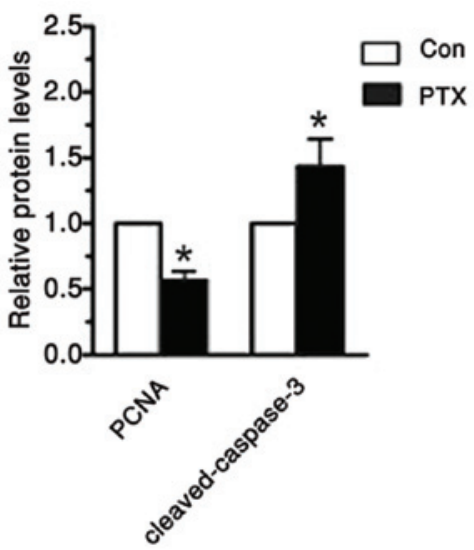

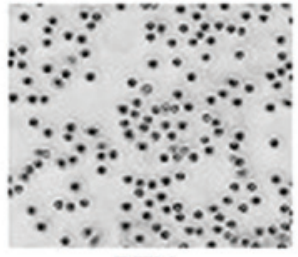

PTX

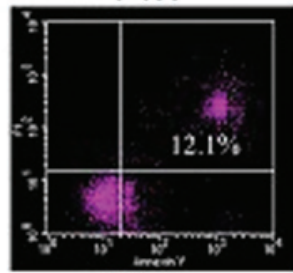

PTX

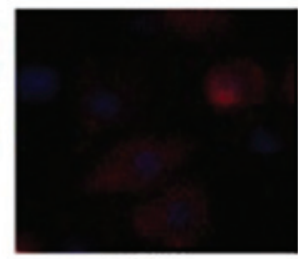

PTX
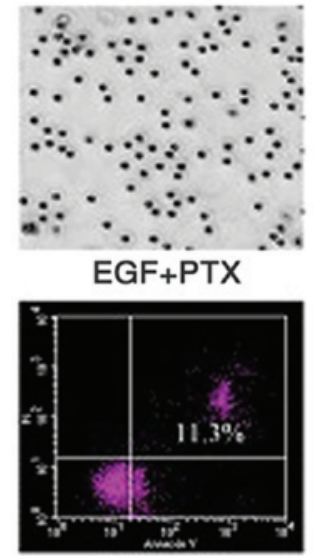

EGF+PTX

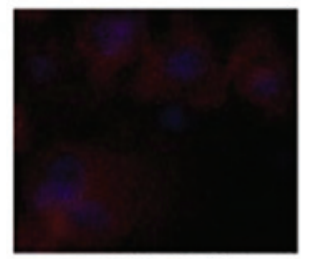

EGF+PTX

Figure 4. PTX suppressed tea8113 cell proliferation and induced its apoptosis. (A) Western blot analysis revealed decreased proliferation marker, proliferative cell nuclear antigen, and enhanced cleaved-caspase-3 levels. (B) Microscope, flow cytometry and immunofluorescence were applied to determine cell numbers, apoptosis and epidermal growth factor receptor protein levels. ${ }^{*} \mathrm{P}<0.05$ versus control.

PTX induced tea8113 cell apoptosis. To further explore the effect of PTX in treating oral cavity cancer, tea8113 cell apoptosis was investigated by pretreating cells with $1 \mu \mathrm{g} / \mathrm{ml}$ PTX for $48 \mathrm{~h}$ and staining these cells with Hoechst. As shown in Fig. 2A, enhanced cell apoptosis was determined when the cells were incubated with PTX. To quantify the apoptotic cells, an annexin V-PI kit was used. Flow cytometry revealed that the apoptotic cells treated with PTX were increased by $87 \%$ compared with the control (Fig. 2B).

PTX suppressed EGFR signaling pathway. It has been reported that EGFR is crucial in human oral cancer. Therefore, first the activation of EGFR was analyzed in human oral cancer samples. Using histochemical analysis, the current study determined that EGFR was aberrantly activated in human cancer samples compared with normal tissue (Fig. 3A). To test the effects of PTX on the EGFR signaling pathway, tea8113 cells were first treated with $10 \mathrm{nM}$ EGF for $24 \mathrm{~h}$, which induced abnormal activation of EGFR. Following this, after pre-incubation with EGF, PTX was added in increasing concentrations of $0,0.1,1,5,10 \mathrm{nM}$. Western blot analysis revealed that PTX significantly suppressed EGFR activation in a dose-dependent manner (Fig. 3B). Furthermore, as EGFR was believed to activate MAPK signaling pathways, the protein levels of ERKs, JNKs and p38 MAPKs were also explored following PTX treatment. As shown in Fig. 3C, both the activation and protein expression levels of ERKs, JNKs and p38 MAPKs were reduced when tea8113 cells were exposed to PTX for $24 \mathrm{~h}$ (Fig. 3C). These data suggested that PTX could effectively suppress the three major pathways of EGFR signaling.

PTX treatment inhibited markers of proliferation and induced cell apoptosis. In a previous report, it had been suggested that ERKs regulated proliferation factors, while JNK and p38 MAPKs are involved in cell apoptosis (3). Therefore, this 
study also investigated cell proliferation markers, proliferative cell nuclear antigen (PCNA) and apoptosis related protein, cleaved-caspase-3 in tea8113 cells treated with PTX. As shown in Fig. 4A, western blot analysis demonstrated that the PCNA protein levels were significantly reduced compared with the untreated group. In addition, when these cells were tested with cleaved-caspase-3, the hallmark of apoptosis, caspase-3 was observed to be cleaved in an active form, which finally led to significant cell apoptosis. To investigate whether such effect was achieved through the EGFR signaling pathway, the EGFR signaling pathway was activated with $20 \mu \mathrm{mol} / \mathrm{l}$ EGF (Fig. 4B). For comparison, the tea8113 cells were pre-incubated with $1 \mu \mathrm{g} / \mathrm{ml}$ PTX for $48 \mathrm{~h}$. On observation under a microscope, cell numbers treated with EGF were significantly more than those treated with PTX $(\mathrm{P}<0.05)$. Furthermore, flow cytometry demonstrated an apoptotic rate that was almost 10 times higher when comparing cells treated with PTX to those treated with EGF. To determine the protein levels of EGFR, immunnofluorescence analysis was conducted. The results showed that EGF significantly enhanced EGFR expression while PTX suppressed it, thereby reducing cell proliferation and enhancing cell apoptosis (Fig. 4B). Based on the above data, it can be concluded that PTX may effectively suppress human oral cancer squamous cell proliferation and induce its apoptosis via the EGFR signaling pathway.

\section{Discussion}

In various tumors, EGFR signaling pathway is highly activated and its abnormal activation leads to significant tumor metastasis and cell proliferation in squamous cell carcinoma (13). Therefore, the selective inhibition of the EGFR signaling pathway has become one of the potential therapeutic targets for the future treatment methods for tumors. Paclitaxel has long been used as an antitumor drug, as it can effectively inhibit DNA synthesis and protein transcription (14). Based on this activity, the possible effect and molecular mechanism of PTX were explored in human oral cancer squamous cell line, tea8113.

According to one study, the EGFR signaling pathway predominantly includes ERK1/2, JNK, p38 MAPK signaling pathways (15). ERK $1 / 2$ is an crucial downstream signal transduction molecule of EGFR. Research shows that the inactivated ERK1/2 is located in the cytoplasm and activated ERK1/2 is translocated to the nucleus (16). It will then enhance the expression of certain oncogenes, including proliferation factors (17). Furthermore, the activation of JNK and p38 was hypothesized to inhibit cell apoptosis through the inactivation of caspase-3 $(18,19)$. In the current study, PTX was demonstrated to significantly suppress human oral cancer squamous cell line tea8113 proliferation in a time- and dose dependent manner $(\mathrm{P}<0.05)$. Tumor cells are characterized by evident cell proliferation capability, and PCNA is considered to be a hallmark of cell proliferation $(20,21)$. According to the current study, when tea8113 cells were treated with PTX, the proliferation capability was significantly reduced as PCNA levels were obviously reduced.

Furthermore, the JNK and p38 cascades are also important branches for EGFR signaling pathway (22). The activation of JNK and p38 signaling pathways leads to the formation of abnormal active forms of caspase-3, and aberrant expression of Bcl-2 family, which eventually induces cell apoptosis (23). In the current report, when tea8113 cells were treated with PTX, cell apoptosis was induced. Flow cytometry analysis revealed that PTX enhanced tea8113 cell apoptosis by more than one-fold. Furthermore, western blot analysis revealed that PTX significantly reduced cleaved caspase-3 levels. To investigate whether PTX exerted its effect on cell proliferation and apoptosis via the EGFR signaling pathway, a siRNA targeting EGFR was selected. When EGFR was knocked-down, the protein levels of PCNA and cleaved-caspase-3 were no longer able to be reversed with PTX treatment. These results indicated that PTX may induce human oral cancer squamous cell tea8113 apoptosis through regulating the EGFR signaling pathway.

The effectiveness of PTX can be demonstrated in tissues, when combined with an advanced imaging technique, such as positron emission tomography (PET). PET is one of most commonly used cancer diagnosis instrumentations and is a functional imaging technique for three dimensional cancer diagnosis. Numerous radioactive tracers are used in PET to image cancer in certain tissues. Certain radioactive tracers may be attached to PTX in order to provide three-dimensional imaging and prove the accuracy of PTX targeting and validity of PTX for cancer treatment $(24,25)$.

In conclusion, the present study suggested that PTX may effectively reduce oral cavity cancer proliferation and induce its apoptosis predominantly by inhibiting the EGFR signaling pathway, indicating the potential use of PTX in targeted cancer therapy.

\section{References}

1. Regezi JA, Sciubba JJ and Jordan RC: Oral Pathology: Clinical Pathologic Correlations. 6th edition. Elsevier Health Sciences, St. Louis, MO, 2012

2. Lookingbill DP, Spangler N and Sexton FM: Sexton: Skin involvement as the presenting sign of internal carcinoma. A retrospective study of 7316 cancer patients. J Am Acad Dermatol 22: 19-26, 1990.

3. Yin XM: Signal transduction mediated by Bid, a pro-death Bcl-2 family proteins, connects the death receptor and mitochondria apoptosis pathways. Cell Res 10: 161-167, 2000.

4. Siegel RL, Miller KD and Jemal A: Cancer statistics, 2015. CA Cancer J Clin 65: 5-29, 2015.

5. Petti S and Scully C: Oral cancer knowledge and awareness: primary and secondary effects of an information leaflet. Oral Oncol 43: 408-415, 2007.

6. Liu D, He J, Yuan Z, et al: EGFR expression correlates with decreased disease-free survival in triple-negative breast cancer: a retrospective analysis based on a tissue microarray. Med Oncol 29: 401-405, 2012.

7. Saxena R, Chandra V, Manohar M, et al: Chemotherapeutic Potential of 2-[Piperidinoethoxyphenyl]-3-Phenyl-2H-Benzo(b) pyran in Estrogen Receptor- Negative Breast Cancer Cells: Action via Prevention of EGFR Activation and Combined Inhibition of PI-3-K/Akt/FOXO and MEK/Erk/AP-1 Pathways. PLoS One 8: e66246, 2013.

8. Roberts PJ and Der CJ: Targeting the Raf-MEK-ERK mitogen-activated protein kinase cascade for the treatment of cancer. Oncogene 26: 3291-3310, 2007.

9. Shen Q, Uray IP, Li Y, et al: Targeting the activator protein 1 transcription factor for the prevention of estrogen receptor-negative mammary tumors. Cancer Prev Res (Phila) 1: 45-55, 2008.

10. Li Z, Zhang H, Chen Y, Fan L and Fang J: Forkhead transcription factor FOXO3a protein activates nuclear factor kappaB through B-cell lymphoma/leukemia 10 (BCL10) protein and promotes tumor cell survival in serum deprivation. J Biol Chem 287: 17737-17745, 2012. 
11. Wang C, Song X, Li Y, et al: Low-Dose Paclitaxel Ameliorates Pulmonary Fibrosis by Suppressing TGF-beta1/Smad3 Pathway via miR-140 Upregulation. PLoS One 8: e70725, 2013.

12. Li H, Duan ZW, Xie P, et al: Effects of paclitaxel on EGFR endocytic trafficking revealed using quantum dot tracking in single cells. PLoS One 7: e45465, 2012.

13. Guo YH, Gao FH, Shi J, Yuan HH and Jiang B: [EGFR-ERK signaling pathway down-regulates miRNA-145 in lung cancer cells]. Zhonghua Zhong Liu Za Zhi 35: 187-192, 2013 (In Chinese).

14. Soares AS, Costa VM, Diniz C and Fresco P: Potentiation of cytotoxicity of paclitaxel in combination with Cl-IB-MECA in human C32 metastatic melanoma cells: A new possible therapeutic strategy for melanoma. Biomed Pharmacother 67: 777-789, 2013.

15. Seshacharyulu P, Ponnusamy MP, Haridas D, Jain M, Ganti AK and Batra SK: Targeting the EGFR signaling pathway in cancer therapy. Expert Opin Ther Targets 16: 15-31, 2012.

16. Meloche S and Pouysségur J: The ERK1/2 mitogen-activated protein kinase pathway as a master regulator of the G1- to S-phase transition. Oncogene, 26: 3227-3239, 2007.

17. Li MS, Li PF, Chen Q, Du GG and Li G: Alpha-fetoprotein stimulated the expression of some oncogenes in human hepatocellular carcinoma Bel 7402 cells. World J Gastroenterol 10: 819-824, 2004.

18. Zhang L and Fang B: Mechanisms of resistance to TRAIL-induced apoptosis in cancer. Cancer Gene Ther 12: 228-237, 2005.
19. Kyaw M, Yoshizumi M, Tsuchiya K, Kirima K and Tamaki T: Antioxidants inhibit JNK and p38 MAPK activation but not ERK $1 / 2$ activation by angiotensin II in rat aortic smooth muscle cells. Hypertens Res 24: 251-261, 2001.

20. Paez-Pereda M, Kuchenbauer F, Arzt E and Stalla GK: Regulation of pituitary hormones and cell proliferation by components of the extracellular matrix. Braz J Med Biol Res 38: 1487-1494, 2005

21. O'Connell RM and Baltimore D: MicroRNAs and hematopoietic cell development. Curr Top Dev Biol 99: 145-174, 2012.

22. Pick A and Wiese $M$ : Tyrosine kinase inhibitors influence ABCG2 expression in EGFR-positive MDCK BCRP cells via the PI3 K/Akt signaling pathway. Chem Med Chem 7: 650-662, 2012.

23. Lee DH, Szczepanski MJ and Lee YJ: Magnolol induces apoptosis via inhibiting the EGFR/PI3K/Akt signaling pathway in human prostate cancer cells. J Cell Biochem 106: 1113-1122, 2009.

24. Lowe VJ, Booya F, Fletcher JG, et al: Comparison of positron emission tomography, computed tomography, and endoscopic ultrasound in the initial staging of patients with esophageal cancer. Mol Imaging Biol 7: 422-430, 2005.

25. Schwarz-Dose J, Untch M, Tiling R, et al: Monitoring primary systemic therapy of large and locally advanced breast cancer by using sequential positron emission tomography imaging with $\left[{ }^{18} \mathrm{~F}\right]$ fluorodeoxyglucose. J Clin Oncol 27: 535-541, 2009. 